\title{
Lidil
}

Revue de linguistique et de didactique des langues

$62 \mid 2020$

Recherches actuelles en didactique du lexique : avancées, réflexions, méthodes

\section{Des graphes lexicaux pour donner à voir des motifs de confusion entre unités lexicales?}

Can Lexical Graphs Show Sources of Lexical Confusion as Visual Patterns?

\section{Veronika Lux-Pogodalla}

\section{(2) OpenEdition}

\section{Journals}

Édition électronique

URL : http://journals.openedition.org/lidil/7973

DOI : $10.4000 /$ lidil. 7973

ISSN : 1960-6052

Éditeur

UGA Éditions/Université Grenoble Alpes

Édition imprimée

ISBN : 978-2-37747-226-0

ISSN : $1146-6480$

Référence électronique

Veronika Lux-Pogodalla, "Des graphes lexicaux pour donner à voir des motifs de confusion entre unités lexicales ? 》, Lidil [En ligne], 62 | 2020, mis en ligne le 03 novembre 2020, consulté le 05 novembre 2020. URL : http://journals.openedition.org/lidil/7973 ; DOI : https://doi.org/10.4000/lidil. 7973

Ce document a été généré automatiquement le 5 novembre 2020

(C) Lidil 


\title{
Des graphes lexicaux pour donner à voir des motifs de confusion entre unités lexicales?
}

\author{
Can Lexical Graphs Show Sources of Lexical Confusion as Visual Patterns?
}

\author{
Veronika Lux-Pogodalla
}

\section{Remerciements}

Merci à mes collègues N. Gader, S. Ollinger et A. Polguère pour leurs relectures et leurs encouragements; merci aux relecteurs pour leurs commentaires constructifs qui ont permis d'améliorer une première version de cet article. Les erreurs restent miennes, cela va de soi.

\section{Introduction}

1 Le travail présenté ici a été mené sur une assez longue période durant laquelle se développaient en parallèle dans mon équipe :

1. le Réseau lexical du français (Gader, Lux-Pogodalla \& Polguère, 2012 ; Lux-Pogodalla \& Polguère 2011 ; Polguère \& Sikora, 2013);

2. un logiciel de visualisation de réseaux/graphes lexicaux, Spiderlex ${ }^{1}$ (ollinger, Polguère, Chudy \& Gaume, 2020);

3. une première collaboration avec des enseignants du secondaire, A. et $O$., qui nous ont sollicités pour notre expertise en lexicologie et lexicographie. Ils avaient établi une liste de " couples de mots souvent confondus par (leurs) élèves", des lycéens de seconde (16 ans environ) : sens et direction, milieu et centre, relation et rapport, tour et révolution, figure et dessin.

Étonnamment pour nous, le caractère très polysémique des mots en cause et le fait que ces mots peuvent parfois être utilisés l'un pour l'autre, comme des synonymes (ex. Il n'y a aucune relation entre ces deux événements. Il n'y a aucun rapport entre ces deux événements) étaient loin d'être des évidences pour nos partenaires enseignants. Par exemple, les enseignants $\mathrm{A}$. et $\mathrm{O}$. ont exprimé de l'indignation, de l'étonnement ou de l'amusement face aux erreurs de leurs élèves, mais jamais ils ne les ont mises en relation avec des 
caractéristiques propres au lexique (polysémie, synonymie, etc.). Ils énonçaient leur constat avec les termes très généraux de " pauvreté du vocabulaire » et de " manque de précision dans l'utilisation des mots » et les explications avancées se limitaient aussi à des généralités (ex. Nos élèves ne lisent plus). Cela nous a convaincue de la nécessité d'une formation minimale en lexicologie de nos partenaires enseignants. Nous rejoignons ici les conclusions de Anctil (2012), Grossmann (2011), Polguère (2004) et Tremblay (2009).

Par ailleurs, nous avions supposé que, quotidiennement exposés aux erreurs des élèves, A. et $\mathrm{O}$. en avaient une connaissance fine. Or en tant qu'enseignants, ils n'ont ni le temps ni le recul nécessaires pour construire une telle expertise, leur priorité étant d'agir en fonction des événements dans la classe pour poursuivre leur activité didactique. Ainsi, la liste de "couples de mots souvent confondus par (les) élèves" constituait une description insuffisante pour identifier précisément les unités lexicales en cause, mais nous n'avons pas pu avoir accès aux contextes d'apparition de ces confusions, A. et $\mathrm{O}$. n'ayant pas jugé intéressant de les conserver. Cette expérience, plus radicalement que n'importe quelle lecture, nous a convaincue de la nécessité d'avoir un accès direct aux productions linguistiques des élèves et de constituer un corpus ainsi que l'a fait Anctil (2011).

4 Finalement, notre première expérience du terrain nous a entrainée dans une recherche exploratoire sur l'utilisation des graphes lexicaux pour analyser quelques erreurs lexicales, dont nous présentons ici les premiers résultats. Nous empruntons à Anctil (2011) sa définition de l'erreur lexicale (section 2.2.1) et situons dans sa typologie, les erreurs que nous décrivons. En l'absence de données de terrain exploitables, nous avons choisi de nous focaliser sur des confusions entre un sens « ordinaire » et un sens spécialisé mathématique décrits dans Baruk (1995). Ce choix nous amène dans le champ de recherche très actif de la didactique de la langue, et plus particulièrement, du lexique, en mathématiques (section 2.2.2).

5 Les données présentées dans la section 3 nous conduisent (1) à décrire dans le RL-fr des couples de mots confondus par les apprenants puis (2) à extraire du RL-fr et à visualiser les graphes correspondant à ces descriptions. Nous explorons ainsi des zones du lexique dont l'apprentissage a occasionné des erreurs, en cherchant d'éventuels patrons (ou «motifs ») caractéristiques de ces zones.

6 À long terme, nous espérons contribuer (1) à une meilleure compréhension de certaines erreurs lexicales, en identifiant et en caractérisant, les zones du lexique où elles risquent d'apparaître ; (2) à la prévention de ces erreurs par un meilleur enseignement du lexique. Notre souhait est de travailler à la réalisation de cet objectif en partenariat avec des enseignants suffisamment formés en lexicologie pour exploiter le RL-fr.

\section{Notions fondamentales, état de l'art}

7 Nous présentons ici les armes et bagages avec lesquels nous nous sommes lancée dans les analyses des erreurs lexicales exposées dans la section 3. Nos armes sont celles de la LEC, la Lexicologie explicative et combinatoire (Mel'čuk, Clas \& Polguère, 1995) qui constitue le volet lexicologique de la Théorie Sens-Texte (Mel'čuk, 1997; Milićević, 2006). Plus précisément, nous nous situons dans la lexicologie des réseaux lexicaux (Polguère, 2009). Nous avons choisi quelques bagages dans la littérature sur l'analyse d'erreurs lexicales. 


\subsection{Des unités lexicales aux graphes lexicaux}

8 Les notions fondamentales indispensables au lecteur pour comprendre le présent article sont expliquées dans le manuel de Polguère (2016). Nous en donnons ici une présentation brève et très simplifiée.

9 Au centre de notre travail, se trouve l'unité lexicale (aussi appelée lexie). Celle-ci (ex. TORTUE I) exprime un sens particulier donné qui peut faire l'objet d'une définition lexicographique (ex. 'animal qui...') ; elle se caractérise aussi par une combinatoire propre.

10 Le vocable est l'unité linguistique potentiellement polysémique. Un vocable (ex. TORTUE) regroupe des unités lexicales (ex. TORTUE I, TORTUE II) partageant un même signifiant et liées entre elles par des liens de copolysémie (Polguère, 2018). Le vocable qui ne regroupe qu'une seule unité lexicale est un vocable monosémique (ex. PANGOLIN). Dans cet article, les vocables sont écrits en petites capitales, les unités lexicales sont aussi notées en petites capitales et sont numérotées quand elles relèvent d'un vocable polysémique ${ }^{2}$.

11 Notre activité lexicographique ne consiste pas à rédiger des articles de dictionnaires décrivant les vocables et les unités lexicales, mais à tisser un réseau lexical (Polguère, 2014a, 2014b), le Réseau lexical du français (RL-fr).

12 Les nœuds du RL-fr sont des unités lexicales (ex. toRTUe I, toRTUe II, PANGOLin). Pour former un réseau, les unités lexicales sont liées entre elles par des liens orientés de différents types. Les unités lexicales d'un vocable polysémique sont liées entre elles par des liens de copolysémie ${ }^{3}$ (Polguère, 2018). Les unités lexicales sont aussi liées à des unités lexicales relevant (essentiellement) d'autres vocables, par des liens de collocation (ex. intensificateur, verbe support) ou par des liens sémantiques (ex. synonyme, générique, contraire) (Mel'čuk \& Polguère, 2007).

Un réseau lexical comme le RL-fr étant mathématiquement un graphe (Gaume, 2004), nous bénéficions des résultats de travaux mathématiques sur les graphes, par exemple pour l'étude de sa topologie, et d'implémentations informatiques, par exemple pour le visualiser ou pour y rechercher des sous-graphes. Ainsi, le logiciel Spiderlex nous permet de faire des recherches dans le RL-fr et de visualiser les résultats sous forme de graphes. La figure 1 montre un extrait du RL-fr pour le vocable TORTUE. Nous voyons que ce vocable polysémique contient deux unités lexicales, TORTUE I, qui dénote un animal (ex. Apeurée, la tortue est rentrée dans sa carapace) et TORTUE II qui dénote une personne très lente (ex. Sur la route, il ne dépasse pas les $80 \mathrm{~km} / \mathrm{h}$ : une vraie tortue!) liées par un lien de copolysémie de type métaphore. Même si la figure 1 ne le fait pas apparaitre, tous les liens de copolysémie ont un type, conformément aux propositions de Polguère (2018) ${ }^{4}$.

14 Chacune des deux unités lexicales de notre exemple est liée à des unités lexicales d'autres vocables par des liens sémantiques de différents types. Là encore, même si la figure 1 ne le montre pas, tous les liens sémantiques du RL-fr sont typés en utilisant le système formel des fonctions lexicales (Jousse, 2010; Wanner, 1996) de la LEC. Par exemple, TORTUE I est liée à $\operatorname{LIÈVE}^{1} \mathrm{~A}$, unité lexicale souvent utilisée comme expression contrastive, par un lien sémantique typé avec la fonction lexicale Contr qui exprime précisément cette opposition. L'unité lexicale TORTUE II est liée à LENT I.2 avec 
la fonction lexicale $A_{1}$ Pred qui permet de lier un nom et un adjectif typiquement utilisé comme modificateur.

Figure 1. - Le vocable TORTUE dans le Réseau lexical du français.

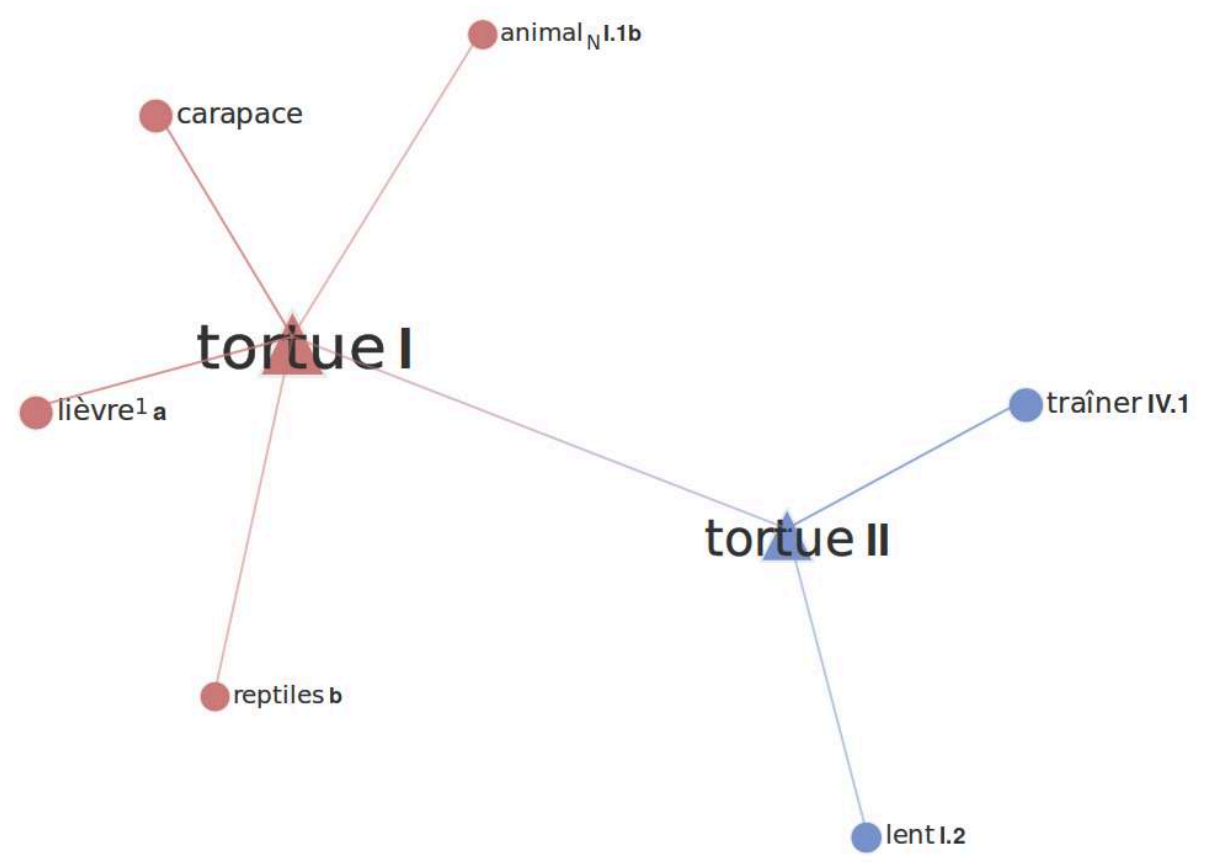

Dans le présent article, par souci de simplicité, nous ne dirons que le strict minimum des quelques types de copolysémie et des quelques fonctions lexicales utilisés.

\subsection{Sur les erreurs lexicales}

\subsubsection{Définition, typologie}

Nous adoptons ici la définition de l'erreur linguistique d'Anctil (2011) comme une «forme linguistique ou combinaison de formes linguistiques qui diffère de ce qu'un locuteur expert aurait selon toute probabilité produit dans un même contexte de production" (p. 70). Le même auteur définit l'erreur lexicale comme une «erreur linguistique qui concerne une propriété inhérente à l'unité lexicale, que cette propriété soit d'ordre formel, sémantique, lexicogrammatical, collocationnel ou pragmatique " (p. 116). Anctil (2011) a analysé 1144 erreurs lexicales relevées dans un corpus d'écrits d'élèves de $3^{\mathrm{e}}$ secondaire (14-15 ans). Il propose une typologie des erreurs lexicales qui repose en premier lieu sur le type de propriété de l'unité lexicale enfreinte (ex. forme, sens, combinatoire), et en second lieu sur l'analyse et la classification des erreurs effectivement observées. Par exemple, dans la classe des erreurs de forme, il définit un type d'erreur lexicale unité lexicale inexistante auquel il recourt pour qualifier l'utilisation de la lexie fictive *apeurant au sens de «inquiétant». Les confusions que nous examinons ici appartiennent à la classe des erreurs lexicales sémantiques d'Anctil : une unité lexicale L1 qui n'a pas le sens présumé par l'élève, est utilisée là où, selon le locuteur expert (ici l'enseignant), l'unité lexicale L2 devrait être utilisée. 
17 La présente recherche est donc focalisée sur une seule classe d'erreur lexicale. Son originalité est d'utiliser les graphes lexicaux pour présenter, de façon graphique, une explication possible de l'erreur. À l'instar d'Anctil, nous cherchons à saisir le lien entre L1 (produite par l'élève par erreur) et L2 (la lexie supposée correcte) à l'intérieur du lexique. Pour cela, dans nos analyses, nous examinons la façon dont L1 et L2 sont liées dans le lexique, en incluant aussi d'autres unités lexicales des vocables polysémiques auxquels L1 et L2 appartiennent et en portant attention aux liens (liens de copolysémie, liens de fonctions lexicales) entre les différentes unités lexicales de ces vocables. Ce contexte est formellement un graphe, extrait du RL-fr.

\subsubsection{Didactique de la langue et mathématiques}

Les travaux sur la maitrise de la langue et les mathématiques sont nombreux, par exemple en France au sein du réseau des IREM (Instituts de recherche sur l'enseignement des mathématiques), qui associe, dans des recherches communes, enseignants du primaire, du secondaire et du supérieur. Parmi d'autres sujets, les spécificités lexicales des mathématiques sont abordées: la synthèse (Eduscol, 2016) souligne que les mathématiques font souvent un usage spécifique de certains noms communs de la langue française et que le sens usuel et le sens pris en mathématiques (par ces noms) ne sont souvent pas totalement étrangers parce qu'il y a eu des allersretours entre les différents contextes d'usage. Les exemples cités incluent les vocables tels que hauteur, base, milieu, centre, fonction, droite, angle, premier, mesure, image, échelle, facteur. Eduscol (2016) recommande des activités sur le lexique pensées de façon interdisciplinaire et souligne la richesse d'un travail étymologique ou lié au champ lexical.

19 Au Québec, des recherches comme celle de Venant, Tremblay et Labrecque (2015) se donnent pour objectif d'ouvrir la voie à des pratiques d'enseignement renouvelées, mettant en relation étroite mathématiques et français. Les auteures mettent en évidence pour quelques vocables polysémique (ex. côTé) les différentes unités lexicales courantes et celles relevant du langage géométrique et explicitent les relations possibles entre celles-ci. Leur hypothèse est que travailler la sémantique des mots de façon globale, en établissant des liens entre sens courants et mathématiques, va permettre de dépasser les blocages lexicaux et les confusions relevées chez les élèves.

20 La recherche exploratoire présentée ici reste en amont de suggestions sur les pratiques d'enseignement. Mais, comme ces auteures, nous montrons, pour des vocables polysémiques, différentes unités lexicales relevant du langage courant pour certaines, d'un langage spécialisé pour d'autres. Pour aller plus loin dans l'explicitation des relations entre celles-ci, nous utilisons les graphes lexicaux.

21 Pour tous les exemples modélisés ici, nous utilisons le Dictionnaire de mathématiques élémentaires de Baruk (1995). Mathématicienne et pédagogue des mathématiques, Baruk est attentive à la polysémie et au parasitage de l'apprentissage de connaissances lexicales spécialisées par des connaissances lexicales générales préexistantes. Dans ce dictionnaire pédagogique, elle anticipe des confusions faisant aussi partie de la liste fournie par A. et 0.5 , par exemple des confusions entre nombre et chiffre, milieu et centre, sens et direction; et ses descriptions sont assez précises pour être modélisées dans le cadre formel de la LEC sans trop de difficulté. Ces deux points font du dictionnaire de Baruk une référence privilégiée dans le présent travail. 


\section{Analyse des erreurs lexicales, visualisation avec des graphes lexicaux}

Dans le cadre théorique de la Lexicologie explicative et combinatoire, nous travaillons depuis 2010 au développement d'un Réseau lexical du français (Gader, Lux-Pogodalla \& Polguère 2012 ; Lux-Pogodalla \& Polguère, 2011 ; Polguère \& Sikora 2013). Toutes les analyses présentées ici ont données lieu à des développements dans le RL-fr et sont visibles en utilisant Spiderlex.

Ci-après, nous donnons pour quelques exemples de confusions lexicales, (1) une analyse appuyée sur Baruk (1995), en particulier pour les sens spécialisés en mathématique ; (2) la visualisation du graphe lexical, extrait du RL-fr, modélisant les unités lexicales en jeu dans la confusion. La visualisation que nous en donnons ici est une simplification de la visualisation possible avec Spiderlex, spécifiquement destinée à nos partenaires enseignants. Au fil des exemples, nous cherchons dans les graphes lexicaux d'éventuels patrons (ou «motifs ») caractérisant des zones du lexique qui prêtent à des confusions lexicales.

\subsection{NOMBRE et CHIFFRE}

La confusion entre NOMBRE et CHIFFRE en mathématiques nous semble un véritable cas d'école.

\subsubsection{Analyse}

En français courant, il existe une lexie CHIFFRE I.1B et une lexie NOMBRE I utilisées comme quasi-synonymes. On dit indifféremment Récite-moi à l'envers les chiffres de 20 à 0 et Récite-moi à l'envers les nombres de 20 à 0 , ou bien Dis un chiffre entre 0 et 100 et Dis un nombre entre 0 et 100 . Selon toute vraisemblance, ce sont des unités lexicales connues des jeunes locuteurs du français.

En mathématiques, la lexie NOMBRE I est très souvent utilisée (ex. Le nombre racine de 2 est connu depuis longtemps et le calcul de sa valeur approchée a été un problème mathématique pendant des siècles). Elle dénote une notion fondamentale et particulièrement difficile à définir. Le dictionnaire de Baruk (1995) n'en propose d'ailleurs pas de véritable définition.

En mathématiques, la lexie CHIFFre I.1B (ex. Dis un chiffre entre 0 et 100) est «illégale ». Seule la lexie chifrRe I.1A (ex. Les numéros de téléphone actuels ont dix chiffres. Comment décomposer un nombre à quatre chiffres?), qui dénote un 'signe qui sert à écrire les nombres', est utilisée. Le caractère illégal de CHIFFRE I.1B apparait à l'école élémentaire, il me semble, et de façon particulièrement nette lorsque les élèves de 8-9 ans (CE2) qui apprennent à écrire les nombres doivent répondre à des questions du type Dans 326 (a) quel est le chiffre des dizaines ? (b) quel est le nombre de dizaines ${ }^{6}$ ?

28 À ce moment précis, l'enseignant s'appuie sur l'opposition NOMBRE et CHIFFRE pour faire comprendre aux élèves la structure des nombres. Nous avons observé des enseignants de CE2 très à l'aise pour introduire l'unité lexicale CHIFFRE I.1A qu'ils définissent comme 'un caractère qui sert à écrire les nombres', faisant un parallèle avec les caractères 
appelés lettres qui servent à écrire les mots. Cependant, ces enseignants agissent comme si ni l'unité lexicale CHIFFRE I.1B ni son lien de synonymie avec NOMBRE I ne préexistaient chez leurs élèves.

Ces derniers ne peuvent donc pas savoir si la nouvelle unité lexicale CHIFFRE I.1A annule et remplace CHIFFRE I.1B qu'ils connaissent déjà, ou bien si elle doit cohabiter dans le lexique avec cette dernière, et comment. Nous avons même eu l'impression que les enseignants ne le savaient pas toujours eux-mêmes et que pour certains d'entre eux, en particulier les plus formés en mathématiques, l'unité lexicale CHIfFRE I.1A était désormais la seule.

Pourtant, n'en déplaise aux mathématiciens, on ne s'exprime pas toujours comme en mathématiques. Dans de nombreux énoncés, la langue courante préfère CHIFFRE I.1B à NOMBRE I à l'inverse de la logique mathématique. Ainsi tel responsable incapable de commenter une baisse de budget de 60 \% sera qualifié de « fâché avec les chiffres ${ }^{7}$ ». Et par souci de pédagogie, après avoir rappelé que « 750000 personnes décèdent chaque année du tabagisme », un journaliste précise : «Pour ceux qui n'aiment pas les grands chiffres, ça fait deux-cents personnes par jour8." Même en mathématiques, des raccourcis sont utilisés dans lesquels l'opposition entre NOMBRE et CHIFFRE est moins nette que ne le laissent croire les exercices de CE2 : ainsi, on dit bien «Un nombre entier est divisible par 3 si et seulement si la somme de ses chiffres est divisible par $3^{9}$ ", alors qu'on pourrait argumenter que l'expression «somme des chiffres» est mathématiquement absurde.

Il existe donc bien deux unités lexicales CHIFFRE I.1B et CHIFFRE I.1A, dont l'une, fait lexicalement remarquable, s'oppose à NOMBRE I tandis que l'autre en est sémantiquement très proche.

\subsubsection{Visualisation}

Ayant modélisé ces faits lexicaux dans le RL-fr, nous pouvons maintenant visualiser dans la figure 2 un graphe extrait du RL-fr qui les contient.

Figure 2. - NOMBRE et CHIFFRE.

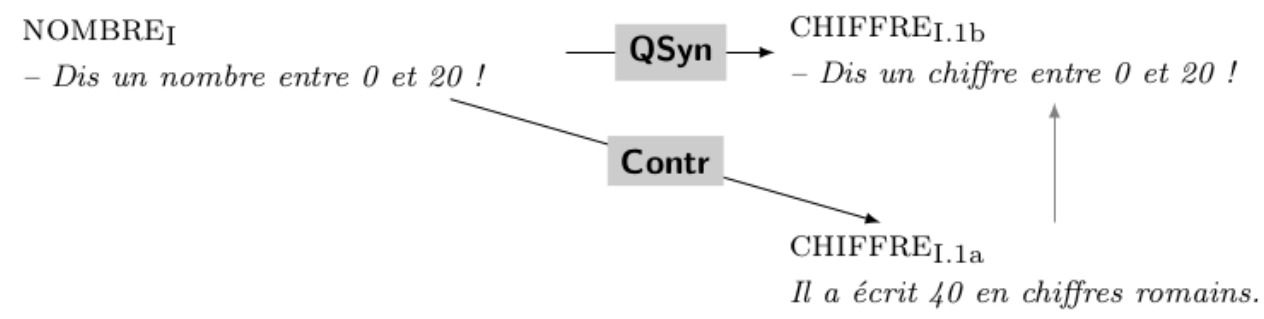

Nous voyons trois unités lexicales distinctes, les trois nœuds du graphe. Le sens de chaque unité lexicale est fixé par un exemple. Les liens entre ces unités lexicales sont figurés dans le graphe par des flèches (ou arcs orientés). Les liens de copolysémie apparaissent en gris. Nous voyons ici un seul lien de copolysémie, entre les unités lexicales CHIFFRE I.1A et CHIFFRE I.1B. Les liens sémantiques apparaissent en noir et sont étiquetés avec des noms de fonctions lexicales. Nous voyons un lien de fonction lexicale QSyn (pour quasi-synonyme) qui modélise la proximité de sens de NOMBRe I et CHIFFRE I.1A 
et un lien de fonction lexicale Contr qui modélise l'opposition de sens entre NOMBRE I et CHIFFRE I.1B.

Nous pouvons visualiser, d'une part, le couple d'unités lexicales courantes connues par le jeune locuteur, NOMBRE I et son quasi-synonyme CHIFFRE I.1B, et, d'autre part, le couple d'unités lexicales sur lequel est focalisé l'enseignant dans un contexte mathématique, NOMBRE I et son contrastif CHIFFRE I.1A.

Enfin, la particularité lexicale que nous avions notée (deux unités lexicales du vocable CHIFFRE dont l'une s'oppose à NOMBRE I, tandis que l'autre en est sémantiquement très proche) correspond à un motif visuel qui est donné dans la figure 3.

Figure 3. - Motif $n^{\circ} 1$

UNITÉ LEXICALE 1

DU VOCABLE 1

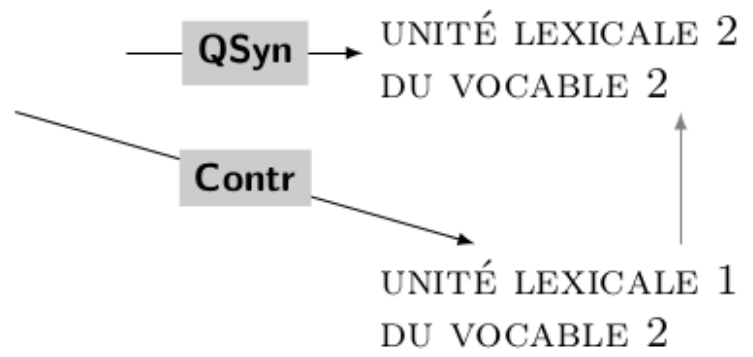

Cela nous amène à nous interroger: est-ce que d'autres erreurs reportées par nos partenaires enseignants apparaissent dans des contextes conformes au même motif?

\subsection{DIRECTION et SENS}

La confusion entre SENS ${ }^{3}$ et DIRECTION est un cas de divergence entre lexique général et lexique mathématique bien identifié et décrit par les didacticiens des mathématiques (Baruk, 1995 ; Loison, Rodriguez \& Sidney, 2001). Cette documentation nous permet très heureusement de limiter notre analyse aux unités lexicales en jeu dans la confusion qui nous intéresse, et d'éviter un examen global de SENS ${ }^{3}$ et DIRECTION qui sont des vocables complexes - leur polysémie est riche (SENS ${ }^{3}$ a aussi des homonymes); le sens de leur unité lexicale de base n'est pas facile à définir.

\subsubsection{Analyse}

Il existe en français courant des lexies DIRECTION I.1 et SENS ${ }^{3}$ I.1A quasi-synonymes. On dit indifféremment «Sur l'A10, en direction de Paris, il y a souvent des embouteillages » ou «Sur l'A10, dans le sens Chartres-Paris, il y a souvent des embouteillages », et «J'ai pris la bonne ligne de métro mais dans la mauvaise direction! » ou «J'ai pris la bonne ligne de métro mais dans le mauvais sens!».

En mathématiques, selon Baruk (1995) «on appelle direction l'ensemble de toutes les droites d'un plan parallèles à une droite donnée » (p.346). Pour Loison, Rodriguez et Sidney (2001), une direction est « une classe d'équivalence de lignes droites selon la relation de parallélisme» (p. 29). Selon ces auteurs, la définition de l'unité lexicale utilisée en mathématiques «correspond assez bien à l'usage courant de ce mot, à condition qu'on n'y mette pas la nuance d'orientation. Ainsi, lorsqu'on parle de direction verticale on ne doit pas préciser, en tant que direction, si c'est de haut en bas ou de bas 
en haut. Ce qui permet de distinguer l'un de l'autre, c'est le sens» (p.29). En mathématiques, des objets pourraient donc se déplacer dans la même direction mais en sens opposé, car « sur une droite quelconque représentant une direction donnée, il y a deux sens de parcours » (Baruk, 1995, p. 346).

Nous avons ici deux lexies spécialisées DIRECTION I.2 et SENS ${ }^{3}$ I.1B dont les sens sont différents de ceux de DIRECTION I.1 et SENS ${ }^{3}$ I.1A. Ces lexies spécialisées ne sont pas synonymes. Elles sont souvent utilisées ensemble et de façon complémentaire dans la définition d'objets mathématiques plus complexes comme les vecteurs (Baruk, 1995); elles peuvent aussi être utilisées isolément - par exemple, il peut être question de sens de parcours sur n'importe quelle ligne, ouverte ou fermée (Baruk, 1995) et pas seulement sur une droite représentant une direction.

\subsubsection{Visualisation}

41 La figure 4 permet de visualiser le graphe comprenant les unités lexicales en jeu dans la confusion analysée.

Figure 4. - DIRECTION et SENS.

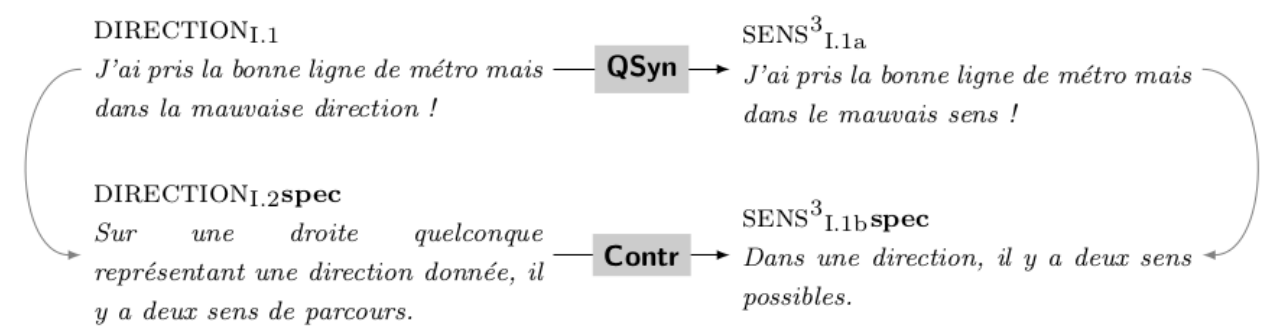

Contrairement au précédent graphe, celui-ci comprend non pas trois mais quatre unités lexicales distinctes. Les deux vocables sont polysémiques et, pour chacun, deux de leurs unités lexicales sont considérées ici. Nous voyons donc, d'une part, les unités lexicales DiRECTION I.1 et DiRECTION I.2, et, d'autre part, les unités lexicales SENS $^{3}$ I.1A et SENS $^{3}$ I.1B reliées deux à deux par un lien de copolysémie.

Néanmoins, comme le graphe précédent, ce graphe contient un lien de fonction lexicale QSyn qui modélise la proximité de sens entre les unités lexicales DIRECTION I.1 et SENS ${ }^{3}$ I. 1A ainsi qu'un lien de fonction lexicale Contr qui modélise l'opposition de sens entre les unités lexicales spécialisées DIRECTION I.2 et SENS ${ }^{3}$ I.1B.

Comme dans le sous-graphe précédent, nous pouvons visualiser deux couples d'unités lexicales : d'une part, le couple d'unités lexicales courantes probablement connues par le jeune locuteur, DIRECTION I.1 et son quasi-synonyme SENS ${ }^{3}$ I.1A, et, d'autre part, le couple d'unités lexicales introduites en cours de mathématique, DIRECTION I.2 et son contrastif SENS ${ }^{3}$ I.1B.

Nous voyons sur les figures 3 et 5 que les motifs pour les couples DIRECTION et SENS d'une part, NOMBRE et CHIFFRE d'autre part, sont semblables à un nœud près. 
Figure 5. - Motif $n^{\circ} 2$

UNITÉ LEXICALE 1

DU VOCABLE 1

\section{UNITÉ LEXICALE 2 \\ DU VOCABLE 1}

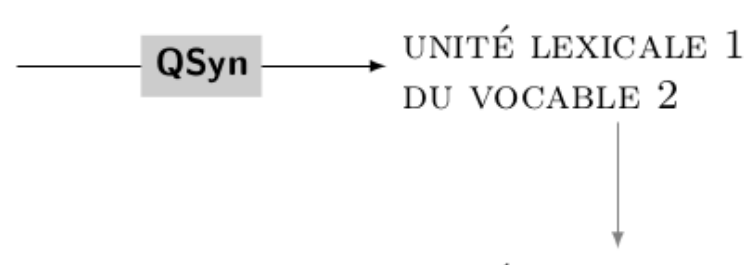

Contr
UNITÉ LEXICALE 2

DU VOCABLE 2

\subsection{MILIEU et CENTRE}

La confusion entre MILIEU et CENTRE en mathématiques se conçoit aisément.

\subsubsection{Analyse}

En effet, il existe en français courant des lexies MILIEU I.1A et CENTRE I.1A quasi synonymes (Picoche \& Rolland, 2002, p. 561-563). Ainsi, les phrases La statue est au milieu de la place et La statue est au centre de la place expriment à peu près le même sens. En mathématiques, les lexies CENTRE I.1B (ex. 1) et MILIEU I.1B (ex. 2) sont utilisées et paraissent, de prime abord, dénoter comme leurs homologues courantes 'la partie d'un objet la plus éloignée des bords de celui-ci' :

1. Voici une méthode géométrique permettant de trouver le centre d'un cercle.

2. Comment calculer les coordonnées du milieu d'un segment dans un repère donné à partir des coordonnées des deux extrémités de ce segment?

Néanmoins, la combinatoire des deux lexies utilisées en mathématiques est plus restreinte : comme l'écrit Baruk (1995) : "Le mot milieu est strictement réservé, en mathématiques, à un point qui est à égale distance des extrémités d'une portion de ligne, et en particulier d'une portion de ligne droite, c'est-à-dire d'un segment " (p. 717).

Aussi, les deux lexies utilisées en mathématique ne sont pas synonymes. Il n'est pas légal de parler du centre d'un segment; il n'est pas légal non plus de parler de milieu d'un cercle ou de milieu d'un rectangle: le " point situé à égale distance de tous les points d'un cercle» est son centre, le point d'intersection des diagonales d'un rectangle est son centre (Baruk, 1995).

\subsubsection{Visualisation}


Figure 6. - MILIEU et CENTRE.

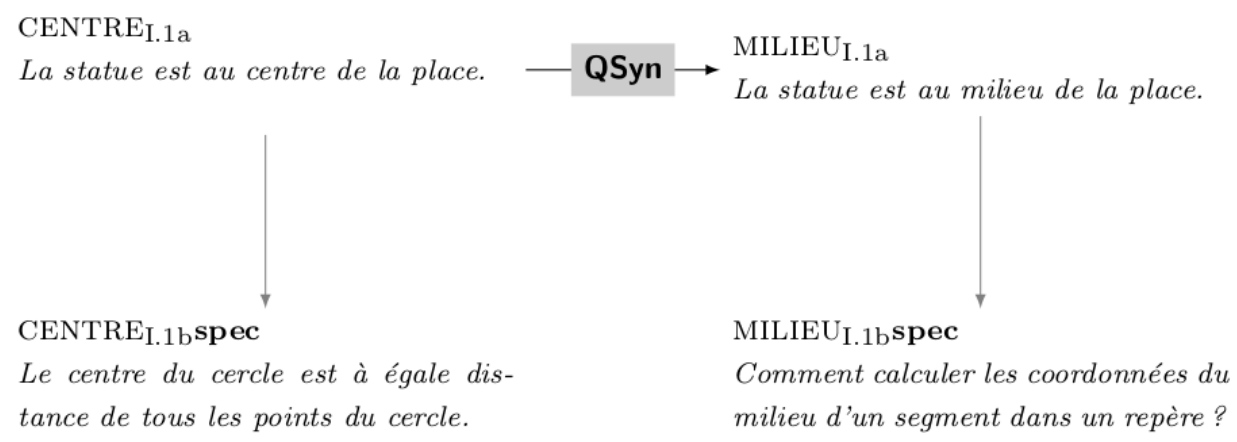

Comme le précédent, ce sous-graphe comprend quatre unités lexicales distinctes : deux fois deux unités lexicales relevant de deux vocables polysémiques différents. Nous voyons donc d'une part les unités lexicales MILIEU I.1A et MILIEU I.1B, d'autre part les unités lexicales CENTRE I.1A et CENTRE I.1B, chaque paire étant reliée par un lien de copolysémie.

51 De même, ce sous-graphe contient également un lien de fonction lexicale QSyn qui modélise la proximité de sens entre les unités lexicales MILIEU I.1A et CENTRE I.1A. Nous voyons un couple d'unités lexicales, MILIEU I.1A et son quasi-synonyme CENTRE I.1A, courantes en français et probablement connues par le jeune locuteur.

Contrairement à ce qui était le cas dans les deux sous-graphes précédents, il n'existe pas de lien d'opposition explicite entre les unités lexicales MILIEU I.1B et CENTRE I.1B : c'est l'absence d'un lien de quasi-synonymie qui déroute ici l'apprenant.

Nous voyons sur les figures 7 et 5 que les motifs pour les couples MILIEU et CENTRE d'une part, DIRECTION et SENS d'autre part, sont semblables à un lien près.

Figure 7. - Motif $n^{\circ} 3$.

UNITÉ LEXICALE 1

DU VOCABLE 1

UNITÉ LEXICALE 2

DU VOCABLE 1

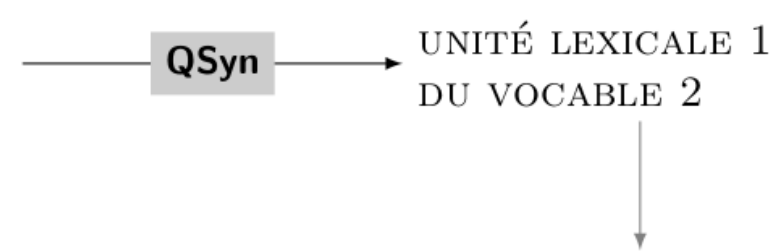

UNITÉ LEXICALE 2

DU VOCABLE 2

\section{Conclusion et perspectives}

Ces analyses nous montrent d'abord que, contrairement à ce que disent nos partenaires enseignants A. et 0 ., les élèves ne confondent pas CHIFFRE et NOMBRE, mais CHIFFRE I.1A et CHIFFRE I.1B. Ils ne confondent pas SENS et DIRECTION, mais SENS ${ }^{3}$ I.1A et SENS ${ }^{3}$ I.1B ou DIRECTION I.1 et DIRECTION I.2, etc. La confusion concerne toujours deux unités d'un vocable polysémique (deux copolysèmes), que l'élève n'a pas correctement identifiées comme distinctes. 
Existe-t-il des types de copolysémie particulièrement difficiles à saisir? Pour les exemples examinés, plusieurs didacticiens des mathématiques ont décrit très en détail les unités lexicales posant difficulté. Avec un plus grand nombre d'exemples, nous souhaiterions essayer de caractériser ces difficultés sur le plan lexicographique et voir si certains types de liens de copolysémie de notre typologie (ex. lien de spécialisation) sont plus souvent en cause que d'autres (ex. lien de métaphore).

Par ailleurs, dans tous les exemples examinés, la confusion entre deux unités lexicales et l'emploi de l'une au lieu de l'autre nous sont révélés par l'utilisation erronée d'une troisième unité lexicale qui est quasi synonyme l'une mais non de l'autre. Cela suggère que l'existence de liens sémantiques différents pour différentes unités lexicales de vocables polysémiques pourrait être exploitée dans l'enseignement pour caractériser de façon contrastive ces différentes unités lexicales et prévenir les confusions. Pour le lexique de la géométrie, Venant, Tremblay et Labrecque (2015) donnent quelques suggestions pratiques dans ce sens.

Enfin, il est remarquable que les graphes modélisant nos analyses nous donnent parfois aussi à voir ce qui cause l'étonnement (fig. 8) des enseignants face à l'erreur : l'élève exploite un lien de quasi-synonymie existant entre les unités lexicales qu'il utilise (par erreur), alors que c'est un lien sémantique de contrastivité ou d'antonymie qui lie les unités lexicales que l'élève aurait dû utiliser. Pour l'enseignant, focalisé sur ces dernières, l'erreur apparaît donc avec un certain éclat.

Figure 8. - Motif de la cause de l'étonnement.

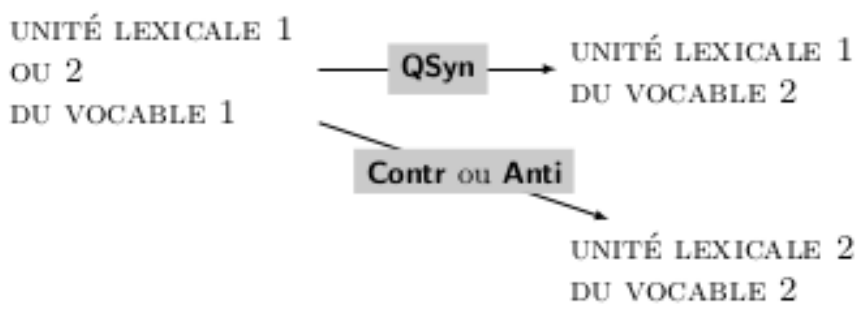

58 C'est un résultat inattendu de ce travail et dans le futur nous aimerions explorer ce dernier motif. Dans la continuité des travaux de thèse d'ollinger (2014) sur l'analogie, il s'agirait notamment de rechercher dans le Réseau lexical du français des sous-graphes conformes à ce motif puis d'étudier les particularités des ensembles d'unités lexicales ainsi identifiés, en se demandant s'ils présentent systématiquement des difficultés d'apprentissage.

Illustrons avec un exemple (non mathématique) cette perspective qui semble peut-être très théorique au lecteur. Ainsi, les unités lexicales dans la figure 9 obéissent au motif de la figure 8 moyennant certaines généralisations sur les liens sémantiques (par exemple, en considérant non seulement la synonymie mais aussi la méronymie). 
Figure 9. - WEEK-END et SEMAINE.

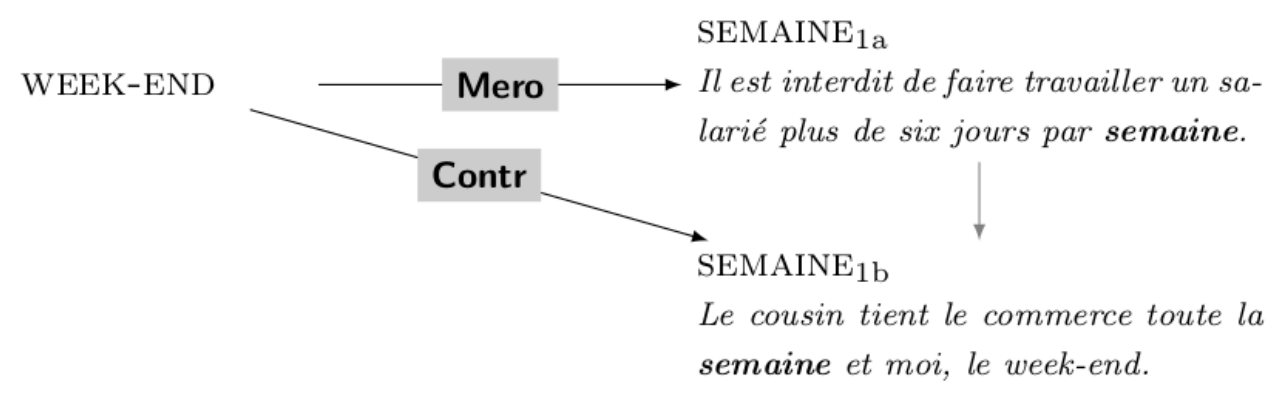

Nous ne savons si ces unités lexicales posent des difficultés aux apprenants, mais on peut s'étonner de la cohabitation dans le lexique d'une unité lexicale SEMAINE dénotant une période de 5 jours et contrastant avec l'unité lexicale WEEK-END et une autre unité lexicale SEMAINE dénotant une période de 7 jours qui inclut le week-end.

Plus généralement, le travail présenté ici était une première tentative d'utiliser une version vulgarisée de notre modèle lexical et une version simplifiée de sa visualisation pour communiquer avec nos partenaires enseignants. Malheureusement, l'interruption brutale de ce premier partenariat pour des raisons extrascientifiques a prématurément mis fin à l'expérience.

Pourtant, les échanges avec A. et $\mathrm{O}$. nous auront finalement aidés à concevoir un programme sur mesure lorsque, dans un partenariat récent, nous avons pu mettre en place une formation pour six enseignants de cycle 3. La polysémie, l'unité lexicale, les liens sémantiques et les liens de collocations entre unités lexicales sont naturellement des thèmes abordés.

Actuellement, alors que nous en arrivons, enfin, à l'exploration de graphes lexicaux avec nos partenaires enseignants, nous utilisons tout naturellement ce que le travail exploratoire présenté ici a bien permis de mettre au point : une visualisation simplifiée des graphes lexicaux ne nécessitant de maitriser parfaitement ni les fonctions lexicales ni les types de copolysémie, et focalisée sur des sous-ensembles spécifiques d'unités lexicales en jeu dans des difficultés lexicales particulières. La présentation de ce travail fera l'objet d'une future publication pour mieux lier les hypothèses et réflexions théoriques et didactiques présentées dans le présent article à un contexte de formation continue des enseignants sur le lexique en général et les lexiques disciplinaires.

\section{BIBLIOGRAPHIE}

ANCTIL, Dominic. (2011). L'erreur lexicale au secondaire : analyse d'erreurs lexicales d'élèves de $3^{e}$ secondaire et description du rapport à l'erreur lexicale d'enseignants du français. (Thèse de doctorat). Université de Montréal, Montréal. 
ANCTIL, Dominic. (2012). Portrait des erreurs lexicales d'élèves de $3^{\mathrm{e}}$ secondaire en production écrite et proposition de pistes didactiques. Pratiques, 155-156, 7-30. <https://doi.org/10.4000/ pratiques.3447>.

BARUK, Stella. (1995). Dictionnaire de mathématiques élémentaires (2e éd.). Paris : Seuil.

Eduscol. (2016). Mathématiques et maîtrise de la langue. Disponible en ligne sur <https:// cache.media.eduscol.education.fr/file/[...]math_maitr_lang_N.D_600996.pdf> (consulté le 2 juin 2020).

GADER, Nabil, LuX-Pogodalla, Veronika \& Polguère, Alain. (2012). Handcrafting a Lexical Network with a Knowledge Based Graph Editor. Dans M. Zock \& R. Rapp (dir.), Proceedings of the Third Workshop on Cognitive Aspects of the Lexicon (CogALex-III) (p. 109-125). Mumbai : The COLING 2012 Organizing Committee.

GAUME, Bruno. (2004). Balades aléatoires dans les petits mondes lexicaux. I3 - Information Interaction Intelligence, 4(2), 39-96.

GrossmanN, Francis. (2011). Didactique du lexique : état des lieux et nouvelles orientations. Pratiques, 149-150, 163-183.

JouSSE, Anne-Laure. (2010). Modèle de structuration des relations lexicales fondé sur le formalisme des fonctions lexicales (Thèse de doctorat). Université de Montréal, Montréal \& Université Paris Diderot (Paris 7), Paris.

LOISON, Philipe, RODRIGUEZ, Danièle \& SIDNEY, François. (2001). Le juste mot en géométrie (Rapport technique). Dijon : IUFM Dijon.

LuX-Pogodalla, Veronika \& Polguère, Alain. (2011). Construction of a French Lexical Network: Methodological Issues. Dans Proceedings of the First International Workshop on Lexical Resources, WoLeR 2011. An ESSLLI 2011 Workshop (p. 54-61). Ljubljana.

MEL'ČUK, Igor. (1997, 10 janvier). Vers une linguistique Sens-Texte. Leçon inaugurale présentée au Collège de France, Chaire internationale, Paris, France.

MEL'ČUK, Igor, CLAS, André \& Polguère, Alain. (1995). Introduction à la lexicologie explicative et combinatoire. Paris / Louvain-la-Neuve : Duculot.

MEL'ČUK, Igor \& POLGUÈRE, Alain. (2007). Lexique actif du français. L'apprentissage du vocabulaire fondé sur 20000 dérivations sémantiques et collocations du français. Bruxelles : De Boeck \& Larcier.

MiLIĆEVIĆ, Jasmina. (2006). A Short Guide to the Meaning-Text Linguistic Theory. Journal of Koralex, $8,187-233$.

OLLINGER, Sandrine. (2014). Le raisonnement analogique en lexicographie, son informatisation et son application au Réseau lexical du français (Thèse de doctorat). Université de Lorraine, Nancy. Oldinger, Sandrine, Polguère, Alain, ChUdy, Yannick \& GAUME, Bruno. (2020). Spiderlex et compagnie. Dans C. Benzitoun, C. Braud, L. Huber, D. Langlois, S. Ouni, S. Pogodalla \& S. Schneider (dir.), Actes de la $6^{e}$ conférence conjointe JEP (31 édition), TALN (27édition), RÉCITAL (22 édition). Volume 4 : Démonstrations et résumés d'articles internationaux (p. 60-63). Nancy.

Picoche, Jacqueline \& RolLAND, Jean-Claude. (2002). Dictionnaire du français usuel. 15000 mots utiles en 442 articles. Bruxelles : De Boeck Duculot.

Polguère, Alain. (2004). Savoir consulter un dictionnaire, c'est bien ; savoir comment le construire, c'est mieux. Québec français, 134, 68-70. 
POLGUÈRE, Alain. (2009). Lexical Systems: Graph Models of Natural Language Lexicons. Language Resources and Evaluation, 43(1), 41-55.

POLGUÈRE, Alain. (2014a). From Writing Dictionaries to Weaving Lexical Networks. International Journal of Lexicography, 27(4), 396-418.

PolguÈre, Alain. (2014b). Principes de modélisation systématique des réseaux lexicaux. Dans Actes de la $21^{e}$ conférence Traitement automatique des langues naturelles (p. 79-90). Marseille.

Polguère, Alain. (2016). Lexicologie et sémantique lexicale : notions fondamentales ( $3^{\mathrm{e}}$ éd.). Montréal : Les Presses de l'Université de Montréal.

POLGUÈRE, Alain. (2018). A Lexicographic Approach to the Study of Copolysemy Relations. Russian Journal of Linguistics, 22(4), 788-820. <https://doi.org/10.22363/2312-9182-2018-22-4-788-820>.

POLGUÈRE, Alain \& SIKORA, Dorota. (2013). Modèle lexicographique de croissance du vocabulaire fondé sur un processus aléatoire, mais systématique. Dans C. Ronveaux, C. Masseron \& C. GarciaDeban (dir.), Enseigner le lexique. Recherches en didactique du français (vol. 5, p. 35-63). Namur : Presses universitaires de Namur.

TREMBLAY, Ophélie. (2009). Une ontologie des savoirs lexicologiques pour l'élaboration d'un module de cours en didactique du lexique (Thèse de doctorat). Université de Montréal, Montréal.

Venant, Fabienne, TRemblay, Ophélie \& LABrecque, Audrey-Anne. (2015). Le lexique au carrefour des mathématiques et du français : pistes pour travailler vocabulaire courant et mathématiques. Bulletin de l'Association mathématique du Québec, LV(3), 68-84.

WANNER, Léo. (1996). Lexical Functions in Lexicography and Natural Language Processing. Amsterdam / Philadelphie : Johns Benjamins Publishing.

\section{NOTES}

1. Cf. <https://lexical-systems.atilf.fr/spiderlex/>.

2. La numérotation des unités lexicales donnée dans cet article est la numérotation du RL-fr au moment de la rédaction.

3. Selon Polguère (2018) : "Polysemy is the property of a vocable to contain more than one sense. [...] Copolysemy between two lexical units L1 and L2 is the formal and semantic relation linking L1 and L2 which conditions their grouping within the same polysemous vocable. » (p. 794) La copolysémie est une relation orientée de L1 vers L2, et typée, le sens de L2 étant relatif au sens de L1 d'une certaine manière (ex. par métaphore, métonymie, spécialisation, etc.).

4. Dans Spiderlex, l'utilisateur peut voir les noms des liens en passant la souris sur ces derniers.

5. Cela ne garantit pourtant pas absolument que les erreurs des élèves de A. et 0 . soient bien celles décrites par Baruk (1995).

6. Réponses attendues : (a) Le chiffre des dizaines est 2 ; (b) Le nombre de dizaines est 32.

7. Cf. < http://jeanpierreguerin.over-blog.com/article-le-maire-est-il-fache-avec-leschiffres-48654020.html> (consulté le 12 février 2018).

8. Cf. < https://www.cnews.fr/sante/2017-03-06/macron-veut-porter-le-prix-du-paquet-decigarettes-10-euros-750358> (consulté le 12 février 2018).

9. Cf. < https://www.lucaswillems.com/fr/articles/1/divisibilite-par-3> (consulté le 12 février 2018). 


\section{RÉSUMÉS}

Les applications pour l'enseignement du lexique ont d'emblée fait partie des objectifs pratiques associés au développement du Réseau lexical du français (RL-fr). Dans la recherche exploratoire relatée ici, en nous appuyant sur les écrits de didacticiens des mathématiques, nous avons analysé quelques cas de confusions entre un sens "ordinaire» et un sens spécialisé mathématique d'un même vocable. Après avoir décrit dans le RL-fr les couples de mots confondus, nous avons extrait du réseau et visualisé les graphes lexicaux correspondant à ces descriptions. Nous faisions l'hypothèse que ces visualisations (1) aideraient nos partenaires enseignants à mieux comprendre les unités lexicales et les liens entre unités lexicales en jeu dans les confusions - ce qui n'a pas pu être vérifié dans cette première collaboration -, (2) qu'elles nous permettraient d'explorer efficacement des zones du lexique dans lesquelles se produisent ces erreurs particulières, en y recherchant d'éventuels patrons (ou « motifs ») caractéristiques - ce qui a été partiellement vérifié.

Teaching has been a target application of the French Lexical Network ever since its inception. In the present preliminary research, we draw upon earlier work from research in the teaching of mathematics and analyze some examples of confusion between a "general" sense and a specialized mathematical sense. We carefully characterized these frequently confused words and extracted the corresponding lexical graphs from the FLN. We hypothesized that these visual representations may (1) help teachers we have partnered with to better understand the behavior of lexical units and their relationships involved in these common mistakes, and (2) highlight areas of the lexical networks where such mistakes occur through the use of characteristic patterns. We observed that both hypotheses were only partially borne out.

\section{INDEX}

Mots-clés : lexicologie, lexicographie, analyse d'erreurs lexicales, partenariat chercheursenseignants

Keywords : lexicology, lexicography, lexical error analysis, partnership between researchers and teachers

\section{AUTEUR}

\section{VERONIKA LUX-POGODALLA}

Laboratoire ATILF - UMR 7118, Université de Lorraine 